\title{
LA JUSTICIA CIVIL DE DOBLE HÉLICE* HACIA UN SISTEMA INTEGRAL DE RESOLUCIÓN DE CONFLICTOS EN SEDE CIVIL
}

\section{CIVIL JUSTICE DOUBLE HELIX TOWARDS AN INTEGRAL SYSTEM OF CONFLICT RESOLUTION IN CIVIL HEADQUARTERS}

Macarena Vargas Pavez ${ }^{* *}$

\section{RESUMEN}

De un tiempo a esta parte asistimos a una paulatina, pero progresiva incorporación de los mecanismos alternativos de resolución de conflictos en el ordenamiento jurídico chileno. Sin embargo, ellos siguen teniendo un papel secundario y marginal. Lo anterior, se contrapone a las tendencias procesales modernas que muestran que una conexión entre el mundo judicial y el mundo de los mecanismos alternativos no solo es posible, sino que indispensable de cara a un sistema de justicia civil accesible y eficiente. El planteamiento de algunas premisas básicas para el diseño de un sistema integral de solución de controversias civiles es el objetivo de este trabajo.

Palabras claves: Justicia civil, proceso judicial, mecanismos alternativos.

AbStRaCt

For a while now, we are witnessing a gradual but progressive incorporation of alternative dispute resolution mechanisms in the chilean legal system.

* La expresión "justicia civil de doble hélice” ha sido propuesta por el profesor Neil Andrews en sus trabajos sobre la justicia civil. La tomo "prestada" para titular este artículo porque ofrece una mirada comprensiva del tema que se aborda.

** Abogada, Universidad Diego Portales. Magíster en Gestión y Políticas Públicas, Universidad de Chile. Doctora en Derecho por la Pontificia Universidad Católica de Valparaíso. Profesora de Derecho Procesal, Universidad Diego Portales. Dirección postal: Avenidad República 112, Santiago. Correo electrónico: macarena.vargas@udp.cl Artículo recibido el 27 de julio de 2017 y aceptado para su publicación el 29 de octubre de 2018. 
However, they still have a secondary and marginal role. The foregoing contrasts with modern procedural trends that show that a connection between the judicial world and the world of alternative dispute resolution is not only possible, but indispensable for an accessible and efficient civil justice system. The approach of some basic premises for the design of an integral system for the solution of civil controversies is the objective of this work.

Key words: Civil justice, judicial process, alternative dispute resolution.

\section{INTRODUCCIÓN}

Tradicionalmente hemos construido los procesos judiciales en forma lineal, como si se tratase de un camino recto que no admite desvíos ni salidas de ninguna especie. Por lo general, el proceso se inicia con una demanda y finaliza -como la mayoría de la doctrina sostiene- con una declaración de mérito, aunque lo correcto sería decir que este no termina, sino hasta la completa ejecución del fallo.

Esta mirada lineal del proceso es la que, a mi juicio, ha impedido la inclusión de los mecanismos alternativos de resolución de conflictos en

196 el marco del sistema de justicia civil. El proceso es uno, los pasos están definidos por la ley y si se siguen desembocaremos en una sentencia judicial que resolverá el asunto controvertido. Desde esta perspectiva, claro, ellos parecen no tener cabida.

Pero sabemos que el proceso judicial no siempre termina en un fallo y que a veces surgen recodos en el camino que obligan a las partes y al juez a tomar decisiones que lo llevan hacia otros derroteros. Ello se observa, por ejemplo, en el caso de un abandono del procedimiento, de un desistimiento de la demanda o de una transacción, instituciones procesales clásicas que modifican el iter regular de un proceso judicial y le ponen término de manera anticipada o "anormal”, como señala alguna parte de la doctrina tradicional.

Detrás de esta mirada subyace la idea de que el proceso judicial es "el" mecanismo (o el más apropiado) para resolver conflictos de relevancia jurídica y que hacerlo por vías distintas a la sentencia judicial sale de la normalidad. Pero hoy las tendencias procesales modernas muestran que la incorporación de otras fórmulas no es solamente es viable, sino que es deseable desde el punto de vista de la accesibilidad y eficiencia del sistema. Comprender el proceso judicial como un constructo flexible y susceptible de experimentar cambios durante su transcurso es la lógica que debiera primar a la hora de analizar la forma de incluir mecanismos colaborativos en la justicia civil.

Este artículo tiene por objetivo plantear algunas premisas acerca del diseño de un sistema integral de resolución de controversias civiles, que 
incluya al proceso judicial como eje articulador del mismo, pero que incorpore, también, mecanismos alternativos de solución de conflictos que puedan conectarse con este en una relación de mutuo equilibrio. El trabajo se enfoca en aquellos asuntos que conocen los tribunales con competencia en materia civil, excluyéndose del análisis aquellos asuntos que siendo de naturaleza civil, como familia o laboral, son resueltos por tribunales especiales.

Para ello, en la primera sección se da cuenta de la difícil relación que en nuestro medio existe entre el proceso judicial y los mecanismos alternativos y como ello se evidencia en el proyecto de reforma a la justicia civil de 2012.

En la segunda sección se describen algunas experiencias comparadas donde se observa una visión comprensiva del sistema de justicia civil que incluye de los mecanismos alternativos, como sucede en Inglaterra y en algunos estados de Estados Unidos. En la tercera sección se plantean algunas premisas a considerar a la hora de diseñar un sistema integral de resolución de controversias civiles, en el marco de procesos de reformas como el que actualmente nuestro país experimenta.

II. ENTRE EL AMOR Y EL ODIO.

LA DIFÍCIL RELACIÓN ENTRE EL PROCESO JUDICIAL

Y LOS MECANISMOS ALTERNATIVOS

En nuestro país la relación entre el proceso judicial y los mecanismos alternativos de resolución de conflictos (en adelante mecanismos alternativos) no ha sido fácil. La hegemonía del proceso y una suerte de superioridad moral que este se ha ganado a lo largo de los siglos lo han transformado, sino en el principal instrumento de resolución de conflictos, en el más relevante.

En materia civil la preeminencia del proceso ha sido casi total desde la dictación del Código de Procedimiento Civil en 1903, pues, aunque dicho cuerpo legal regula también la conciliación intraprocesal, ella no goza de la misma adhesión ni ha mostrado buenos resultados en la práctica ${ }^{1}$. Sin embargo, a partir del nuevo milenio este panorama ha cambiado y se observa una oferta cada vez más amplia de mecanismos alternativos en muy variados ámbitos².

${ }^{1}$ Centro de Estudios de Justicia de las Américas (2011), pp. 23 y 25. En el procedimiento ordinario el porcentaje de causas terminadas por conciliación alcanza al 0,05\% del total y en el procedimiento sumario asciende al 1,2\%. Para mayor información, véase: http://rpc.minjusticia.gob.cl/media/2013/04/Estudio-Trayectorias-Causas-Civiles-enTribunales-Civiles-Santiago.pdf. [Fecha de consulta: 10 de abril de 2018].

${ }^{2}$ Vargas, Macarena y Fuentes (2018), pp. 34-35. 
Expresiones de lo anterior son: la regulación de la mediación para asuntos de familia ${ }^{3}$, daños en salud ${ }^{4}$, relaciones laborales ${ }^{5}$ y conflictos de consumo en materia financiera ${ }^{6}$, por mencionar algunos ejemplos. Lo mismo ocurre con la aprobación hace más de una década de la Ley de Arbitraje Comercial Internacional ${ }^{7}$ y la permanente aplicación del arbitraje interno, ya sea de carácter institucional o ad hoc, constituyendo una manifestación palpable de este último caso, la elaboración por parte de la Corte Suprema de un registro de jueces árbitros para las distintas jurisdicciones del país ${ }^{8}$.

Con todo y, a pesar del auge que estos mecanismos han experimentado en las últimas décadas, siguen teniendo un papel claramente menor en la dinámica procesal. El empalme de los mecanismos alternativos con el proceso judicial ha sido un camino lento y pedregoso que ha debido sortear grandes obstáculos, desde la suspicacia total hacia una aceptación, digamos que limitada y llena de aprehensiones por parte de los operadores del sistema. Se ha discutido muchísimo sobre cómo incorporarlos, quienes deben hacerse cargo de conducirlos y qué valor dar a los acuerdos que allí se alcancen y lo cierto es que -pese a las cambios legales realizados- estos mecanismos siguen representado un papel secundario. Si esto fuese una obra de teatro, el proceso sería, sin lugar a dudas, el protagonista de la historia y los mecanismos alternativos solo actores de reparto.

Lo anterior se observa con nitidez en el proyecto de ley NCPC, el cual de acuerdo con el Mensaje Presidencial, tiene como finalidad ofrecer a la ciudadanía un nuevo sistema procesal civil y

${ }^{3}$ Ley 19968 que crea los Tribunales de Familia publicada en el Diario Oficial el 30 de agosto de 2004. En el año 2008 se dicta la Ley 20286 publicada en el Diario Oficial el 15 de septiembre de 2008, que introducen varias modificaciones al nuevo cuerpo legal, entre ellas el establecimiento de la mediación previa y obligatoria para asuntos de alimentos, cuidado personal y relación directa y regular, manteniendo las restantes materias -en que la mediación está permitida-su carácter voluntario.

${ }^{4}$ Artículo 43, Ley 19966, que establece el Régimen de Garantías en Salud. Allí se regula la mediación obligatoria y previa para conflictos de daños por salud como requisito de procesabilidad de la demanda civil indemnizatoria.

${ }^{5}$ Ley 20087 que sustituye el procedimiento laboral contemplado en el libro v del Código del Trabajo publicada en el Diario Oficial el 3 de enero de 2006 y que contempla el nuevo procedimiento de tutela de derechos fundamentales establece la obligación de la Inspección del Trabajo de llevar a cabo un proceso de mediación. Art. 486 inc. 6.

${ }^{6}$ Ley 19496 de Protección de Derechos de los Consumidores. En el ámbito de consumo de productos o servicios financieros se establece un proceso de mediación de carácter extraprocesal, voluntario y gratuito a cargo de los mediadores acreditados ante el SERNAC.

${ }^{7}$ Ley 19971 de Arbitraje Comercial Internacional publicada en el Diario Oficial el 29 de septiembre de 2004.

${ }^{8}$ Corte Suprema. Auto acordado 128. Instructivo para el registro y designación de jueces árbitros por parte de los tribunales ordinario. 17 de agosto de 2015. Disponible en www.leychile.cl/Navegar?idNorma=1080896 [fecha de consulta: 20 de julio de 2018]. 
"garantizar el acceso a la justicia de todos los ciudadanos y establecer y regular procedimientos adecuados y rápidos para la justa obtención de la tutela solicitada y con la posibilidad de ejecución, cuando ella sea necesaria"9.

Para conseguir estos objetivos la propuesta legislativa introduce varias innovaciones, una de las principales -y más destacadas- es la incorporación de lógica del juicio por audiencias a través de procedimientos orales, inmediatos y públicos. Sin embargo, y pese a la magnitud de las reformas presentadas, uno de los grandes ausentes fueron (y hasta ahora siguen siendo) los mecanismos alternativos ${ }^{10}$. Salvo por la regulación de la conciliación judicial al interior del proceso, que, dicho fuera de paso, es prácticamente idéntica a la contenida en la legislación vigente ${ }^{11}$, el proyecto de ley $\mathrm{NCPC}$ no regula instancias de mediación, arbitraje u otras fórmulas alternativas de solución de controversias en ninguna instancia del proceso o en forma previa a este.

Vale la pena señalar que en este punto el Mensaje Presidencial del referido proyecto de ley es -a lo menos- ambiguo, pues por una parte señala que uno de los ejes centrales que inspiran el nuevo código son precisamente los mecanismos alternativos y que la justicia civil debe ser entendida como un sistema que englobe a todos los mecanismos de resolución de conflictos (el énfasis es nuestro) ${ }^{12} \mathrm{y}$, por otra, sostiene que la regulación de mecanismos alternativos será objeto de una ley especial:

"En esta materia, el Código no regula la existencia de tales mecanismos, ya que aquello será tarea de leyes especiales, estableciéndose como vía externa a la sede judicial”" ${ }^{13}$.

Cuesta entender esta contradicción del Ejecutivo. Por un lado, aboga por un sistema integral de resolución de controversias y, por otro, entrega sin mayores explicaciones la regulación de los mecanismos alternativos a un cuerpo legal distinto, respecto del cual hasta la fecha no se conocen

${ }^{9}$ Mensaje de S. E. el Presidente de la República, con el que se inicia un proyecto de ley que establece el nuevo código procesal civil (2012), p. 17.

${ }^{10} \mathrm{~A}$ la fecha de cierre de este trabajo no se conocía aun la nueva versión del proyecto de ley NCPC en proceso de revisión por parte de una comisión creada en 2018 por el Ministerio de Justicia en los primeros meses de la administración del presidente Sebastián Piñera.

${ }^{11}$ Artículo 280 n. ${ }^{\circ}$ 4. Contenidos de la audiencia preliminar. La única variación que se observa en relación con el llamado a conciliación se refiere a la oportunidad en que este debe efectuarse. La propuesta establece que el juez intentará la conciliación en el marco de la audiencia preliminar, pudiendo si así lo desea citar a una audiencia especialmente para estos efectos en cualquier etapa del proceso.

${ }^{12}$ Mensaje de S. E. el Presidente de la República (2012), p. 17.

${ }^{13}$ Op. cit., pp. 18-19. 
sus lineamientos ni articulado. Si bien no es conditio sine quanon que estos mecanismos se encuentren insertos o normados en los códigos procesales, desde el momento que su regulación se escinde del cuerpo normativo central, cualquier pretensión de globalidad -como la que procura el proyecto de ley NCPC- se ve seriamente frustrada.

A mi juicio, la decisión del Ejecutivo de "separar las aguas" entre los mecanismos alternativos y el sistema de justicia civil solo refuerza una percepción más o menos consolidada en nuestro medio que sitúa a los primeros en un nivel de menor jerarquía (o podríamos decir de "menos linaje") en comparación con el proceso judicial. Ello atenta contra la instalación de una dinámica que podría ser potente y expansiva en términos de acceso a la justicia y de eficiencia del sistema. Su regulación en un código procesal se justifica también por una razón de cultura legal. Aun cuando la regulación normativa no es garantía suficiente de la aplicación y éxito de nuevas instituciones, en países como el nuestro, donde el litigio es considerado el principal medio para resolver los conflictos, las normas representan un significativo papel a la hora de promover cambios en el comportamiento de los actores del sistema.

La "separación de aguas" puede explicarse, entre otras razones, por la falta de claridad sobre cómo conectar los mecanismos alternativos con el proceso judicial. ¿Previos o dentro del proceso? ¿'Voluntarios u obligatorios? $200 \quad$ ¿A cargo de terceros externos al Poder Judicial o dependientes de este? ¿Costeados por las partes, por el Estado o a través de un sistema mixto? Son tantas las opciones que parece que el legislador prefirió mantener el statu quo y dejar para otra ocasión su inserción en el sistema procesal civil.

Algunos dirán que en la actualidad esto no constituye un gran problema, pues la tramitación parlamentaria se encuentra paralizada desde el año 2014, pero me parece que en el marco de una reforma tan sustantiva como la que se pretende -y que el nuevo gobierno del presidente Sebastián Piñera se ha comprometido a retomar ${ }^{14}$ - la ausencia de mecanismos distintos al proceso judicial es sintomático del punto aquí planteado.

${ }^{14}$ La página web del Ministerio de Justicia señala expresamente que dicha repartición se encuentra trabajando "en la reforma al proceso civil, la cual busca acercar la justicia a las personas, con procesos más públicos, transparentes, que resuelva los conflictos con mayor prontitud y en la cual el juez tenga un papel preponderante". Disponible en www.minjusticia.gob.cl/reformas/justicia-procesal-civil/ [Fecha de consulta: 15 de julio de 2018]. En el mismo sitio web se encuentra una nota acerca de la reunión del ministro Hernán Larraín con el Pleno de la Corte Suprema en marzo de 2018 se reseña lo siguiente: "Queremos tener un sistema de trabajo que nos permita avanzar en temas que son de interés común”. En tal reunión, detalló que los ejes de su gestión se enfocarán en fortalecer el acceso a la justicia, transformar el procedimiento civil; procesos rápidos y con mucho uso de la tecnología digital. Disponible en www.minjusticia.gob.cl/ministro-hernan-larraintras-cita-con-pleno-corte-suprema-queremos-tener-un-sistema-de-trabajo-que-nos-permitaavanzar-en-temas-que-son-de-interes-comun/ [Fecha de consulta: 15 de julio de 2018]. 


\section{UN SISTEMA INTEGRAL DE SOLUCIÓN DE CONTROVERSIAS CIVILES ES POSIBLE}

Una visión moderna del sistema de justicia civil debe avanzar hacia el diseño de sistemas de resolución de controversias civiles integrales, flexibles y plurales, capaces de ofrecer a los justiciables respuestas diferenciadas -y donde sea posible- combinar distintos mecanismos (judiciales y alternativos) en distintas etapas del proceso. La tarea de un código procesal civil debe ser la de articular variadas opciones de resolución de conflictos y regular el tránsito coordinado entre cada una de ellas.

Ello se condice con una comprensión amplia del derecho de acceso a la justicia, tal como Mauro Cappelletti y Bryant Garth señalaron hace ya más de veinte $\operatorname{años}^{15}$. El enfoque de acceso a la justicia que estos autores propusieron contempla la creación de nuevos métodos para decidir las reclamaciones jurídicas como, por ejemplo, el arbitraje y la conciliación e, incluso, la incorporación de incentivos económicos para lograr acuerdos fuera del tribunal ${ }^{16}$. Sostienen que este derecho no puede circunscribirse solo a la provisión de servicios de asistencia jurídica para los más pobres, sino que su mejoramiento y expansión pasa también por considerar otros dispositivos que permitan a las personas prevenir y procesar sus conflictos ${ }^{17}$.

Sin embargo, algunos autores se oponen a estas premisas. La profesora inglesa Hazel Genn considera que los mecanismos alternativos y, en particular, la mediación no aumentan el acceso a la justicia, pues esta tiene una base no judicial y, además, no contribuye a la justicia sustantiva porque es necesario que las partes renuncien a sus derechos durante la mediación y se focalicen, en cambio, en resolver el problema ${ }^{18}$. En esta misma línea, vale la pena recordar que décadas atrás el profesor de Yale, Owen Fiss, planteó una fuerte crítica a los mecanismos alternativos. Por distintos argumentos, sostuvo que estos mecanismos socavan el objetivo de los tribunales y de los procesos judiciales con una visión muy optimista de estos mecanismos y muy simplificada de la función de los tribunales ${ }^{19}$.

Con todo, una comprensión amplia, como la que se propone en este documento, postula que el derecho de acceso a la justicia no se agota en el proceso y en la decisión jurisdiccional, sino que incluye otras vías de solución de conflictos de carácter extrajudicial e, incluso, no estatal, como

\footnotetext{
${ }^{15}$ Cappelletti y Garth (1996), $130 \mathrm{p}$.

${ }^{16}$ Op. cit., p. 53.

${ }^{17}$ Op. cit., p. 46.

${ }^{18}$ Genn (2013), p. 409.

${ }^{19}$ Fiss (1984), 28 p.
} 
la justicia indígena, la justicia de paz y los mecanismos alternativos ${ }^{20}$. Esto implica dejar atrás el paradigma de un sistema procesal civil y avanzar hacia un sistema de resolución de conflictos civiles (el énfasis es nuestro).

Una imagen que sirve para ilustrar este enfoque es la que propone el profesor inglés Neil Andrews a través de la idea de la "justicia de doble hélice" ${ }^{21}$. Sostiene que al igual que la molécula del ADN -compuesta por la unión de dos cadenas entrelazadas- el sistema de justicia civil estaría formado, por un parte, por la cadena del proceso ante los tribunales y, por otra, por la cadena de los mecanismos alternativos de resolución de conflictos ${ }^{22}$.

Me parece que esta imagen es elocuente y permite visualizar una relación complementaria y no excluyente entre los mecanismos alternativos y el proceso judicial en una lógica de mutua colaboración que es posible observar en algunas legislaciones comparadas. Allí se han adoptado políticas de justicia tendientes a ampliar el espectro de mecanismos de solución de conflictos civiles (judiciales y alternativos) sin hacerlos competir, intentando resolver las incertidumbres que genera la ausencia de disposiciones legales que regulen la interacción entre ellos y que pongan fin a posibles contradicciones ${ }^{23}$.

Desde esta perspectiva, la vía judicial no desaparece de vista porque las partes hayan presentado un caso a arbitraje o porque hayan comenzado una mediación y viceversa. Por su parte, los tribunales no dejan de alentar el uso de los mecanismos alternativos, porque las partes hayan optado inicialmente por la vía judicial ${ }^{24}$.

La noción de un sistema integral de resolución de conflictos no es algo nuevo y se observa en la legislación de varios países ${ }^{25}$, como sucede en Inglaterra y algunos estados de Estados Unidos. En todos ellos, se advierte una política estatal de avanzar hacia una masificación de los acuerdos voluntarios y de favorecer el uso de los mecanismos alternativos, cada uno de estos sistemas tiene, por supuesto, sus propios énfasis y características.

${ }^{20}$ González (2007). Véase también: Programa de Naciones Unidas para el Desarrollo- PNUD (2005) y Centro de Estudios de Justicia para las Américas (2016).

${ }^{21}$ Andrews (2013), p. 249.

${ }^{22}$ Op. cit., p. 249.

${ }^{23}$ Ibid.

${ }^{24}$ Ibid.

${ }^{25}$ Steffek y Unberath (2013), p. 490. Este libro da cuenta de la realidad de los mecanismos alternativos en doce países, donde se describe y analiza el marco regulatorio y la realidad social en que estos mecanismos son aplicados. 


\section{Algunas notas sobre mecanismos alternativos en el sistema de justicia civil inglés}

En Inglaterra el proceso de incorporación de los mecanismos alternativos $\mathrm{y}$, en particular, de la mediación puede situarse a partir del Informe de "Acceso a la Justicia" de lord Harry Woolf en el año 1994. Este informe es el resultado de una completa revisión a la justicia civil motivada, entre otras razones, por el gasto estatal en asistencia jurídica gratuita y por el aumento de los costos de la justicia penal, que ponía presión sobre los recursos destinados a la justicia civil ${ }^{26}$. Se encomienda entonces a lord Woof la realización de un diagnóstico del sistema de justicia civil y la proposición de reformas que tiendan a dar mayor accesibilidad a menores costos tanto para los litigantes como para el Estado ${ }^{27}$.

Los informes preliminares mostraron que los problemas de costos y demoras, además, de la complejidad misma de la justicia civil estaban vinculados al comportamiento de los abogados y a sus tácticas adversariales. De allí que la propuesta de lord Woolf apuntara hacia a la introducción del case managment y la promoción de acuerdos tempranos entre las partes $^{28}$, recomendándose a los tribunales alentar derechamente el uso de mecanismos alternativos y, una vez hecho esto, a tomar en cuenta la conducta de las partes ${ }^{29}$.

Estas recomendaciones fueron recogidas luego en el Acta de Acceso a la Justicia de 1999 (Access to Justice Act) donde se estableció que las partes antes de buscar ayuda legal debían intentar primero la mediación. Se le otorgó, además, el mismo valor a los acuerdos obtenidos en mediación u otros mecanismos alternativos que los resultados obtenidos ante los tribunales ${ }^{30}$.

Las sugerencias de lord Wolf fueron recogidas también en las Civil Procedure Rules, donde se establece en forma clara y categórica que el proceso judicial debiera ser el último recurso para resolver una controversia jurídica ${ }^{31}$ y que las partes deben considerar si la negociación o cualquier otro mecanismo alternativo les permitiría resolver su conflicto antes de

${ }^{26}$ GenN (2013), p. 398.

${ }^{27}$ Ibid.

${ }^{28}$ Op. cit. p. 401.

${ }^{29}$ LORD Woolf, Her Majesty's Stationary Office (1996)."The Court will encourage the use of ADR al case management conferences and pre-trial reviews, and will take into account whether the parties have unreasonably refused to try or behaved unreasonably in the course of ADR".

${ }^{30}$ GenN (2013), p. 402.

${ }^{31}$ www.justice.gov.uk/courts/procedure-rules/civil/rules/pd_pre-action_conduct. [Fecha de consulta: 3 de abril de 2017]. 
comenzar un proceso judicial e incluso una vez que el proceso ya se ha iniciado $^{32,33}$.

Desde esta perspectiva, los tribunales pueden sugerir a las partes o alentar el uso de los mecanismos alternativos, pero no pueden imponer obligatoriamente su utilización. Sin embargo, están facultados para suspender el procedimiento con el fin de darles cabida e, incluso, pueden sancionar en costas a la parte que no concurre a una mediación sin una razón justificada ${ }^{34}$. La sanción por esta conducta se determinará en función de distintos criterios como la naturaleza de las disputas, el mérito del caso, si se han intentado otros mecanismos alternativos y si estos tienen posibilidades razonables de éxito, entre otros ${ }^{35,36}$.

Por su parte, la mediación se puede combinar con el proceso judicial, con el arbitraje e, incluso, con otros mecanismos alternativos a través de un sistema de resolución de conflictos con cláusulas contractuales de "niveles múltiples" que permite ir pasando de un mecanismo a otro ${ }^{37}$. Así, en primer lugar, se intenta una negociación directa entre partes, si ella fracasa se contempla un proceso de mediación obligatorio y si el conflicto sigue sin resolverse se acude al proceso judicial ${ }^{38}$. En algunos casos, estas cláusulas pueden establecer que la mediación sea una etapa obligatoria antes de iniciar un proceso judicial, pero también podría ser una exigencia 204 previa al inicio de un arbitraje ${ }^{39}$.

El fortalecimiento de estos mecanismos en Inglaterra se observa también en materia arbitral, cuya regulación sigue a la Ley Modelo de la UNCITRAL y cubre una amplia gama de asuntos ${ }^{40}$. En el ámbito internacional, el arbitraje comercial goza de gran aceptación por la flexibilidad y

${ }^{32}$ CRP. Pre-action protocols.

${ }^{33}$ LORD Woolf, Her Majesty's Stationary Office (1996): "Litigation should be a last resort. As part of a relevant pre-action protocol or this Practice Direction, the parties should consider whether negotiation or some other form of ADR might enable them to settle their dispute without commencing proceedings. 9. Parties should continue to consider the possibility of reaching a settlement at all times, including after proceedings have been started. Part 36 offers may be made before proceedings are issued".

${ }^{34}$ Andrews (2013), p. 257

${ }^{35}$ Op. cit., p. 261.

${ }^{36}$ CRP. Pre-action protocols: "If proceedings are issued, the parties may be required by the court to provide evidence that ADR has been considered. A party's silence in response to an invitation to participate or a refusal to participate in ADR might be considered unreasonable by the court and could lead to the court ordering that party to pay additional court costs". Disponible en www.justice.gov.uk/courts/procedure-rules/ civil/rules/pd_pre-action_conduct. [Fecha de consulta: 3 de abril de 2017].

${ }^{37}$ Andrews (2013), p. 233.

${ }^{38}$ Ibid.

${ }^{39}$ Ibid.

${ }^{40}$ Andrews (2013), p. 227. 
privacidad del proceso, por la oportunidad que se da a las partes de elegir al árbitro (los cuales pueden no ser abogados) y por la posibilidad de una ejecución rápida ${ }^{41}$. En el ámbito interno, las partes pueden acordar otra modalidad de arbitraje que opera a través de la llamada cláusula Med-Arb, en virtud de la cual si la mediación fracasa el mediador cambia su papel y se convierte en árbitro con la facultad de tomar una decisión vinculan$\mathrm{te}^{42}$. También puede suceder al revés, que estando dentro de un proceso de arbitraje se desarrolle una mediación, permitiéndole al árbitro actuar como mediador, esto es, lo que se ha dado en llamar un árbitro con "dos sombreros" $"$.

Para cerrar este apartado diremos que Hazel Genn tiene una opinión negativa acerca de la expansión que estos mecanismos han tenido en los últimos años en Inglaterra, especialmente de la mediación, sosteniendo que se han posicionado y han alcanzado un gran auge sobre la base de una retórica antiderecho (anti-law) que ha llevado al decaimiento de la justicia civil ${ }^{44}$.

\section{Algunas notas sobre mecanismos alternativos en el sistema de justicia civil de Estados Unidos}

Un enfoque integral del sistema de justicia civil se observa en Estados Unidos a partir del modelo de Multi door Courthouse (en adelante Tribunal Multi-puertas) que se aplica desde hace décadas en varios estados de Estados Unidos. Tiene como finalidad determinar el método más apropiado de resolución de conflictos y con menores costos de tiempo y dinero tanto para los tribunales como para las partes. Busca ofrecer a las personas un fácil acceso a la justicia, reducir las demoras y proveer enlaces a servicios relacionados, abriendo nuevas opciones para que los conflictos sean solucionados ${ }^{45}$.

Se parte de la base de que los conflictos presentan distintos niveles de complejidad y de adversarialidad y que, además, avanzan a distintos ritmos, de modo que no es posible ofrecer a los afectados solo una vía de solución. Por ello, se hace necesario explorar desde los tribunales formas alternativas de resolución de conflictos y establecer un sistema de asigna-

${ }^{41}$ Andrews (2013), p. 229.

${ }^{42}$ Op, cit., p. 234.

${ }^{43}$ Aguirrezábal, Pérez Ragone y Vargas (2011), p. 365.

${ }^{44}$ Genn (2013), p. 409.

${ }^{45}$ Un detallado desarrollo del funcionamiento de los tribunales multipuertas en Estados Unidos puede verse en el artículo de Cristian Riego y Ricardo Lillo, donde se explican los inicios del modelo, proceso de desarrollo y sus resultados. RieGO y LiLLO (2014), pp. 385-417. 
ción a ciertos métodos o "puertas", según las particularidades del caso ${ }^{46}$. La determinación de la "puerta" apropiada para resolver un conflicto la hace un equipo de especialistas que asiste a las partes para encontrar la mejor alternativa. A este servicio se puede acceder sin necesidad de una solicitud formal, incluso en forma telefónica, y no se exige haber ingresado el caso al sistema judicial ${ }^{47}$.

A diferencia de la reforma inglesa de la década de 1990, el modelo de Tribunal Muti-puertas no es el resultado de un proceso de revisión del sistema de justicia civil, sino que surge como consecuencia de una discusión académica acerca de las causas de insatisfacción de la ciudadanía con el sistema judicial $^{48}$. En efecto, en 1976 Warren E. Burger, juez de la Corte Suprema de Justicia, organizó la famosa Conferencia Roscoe Pound, donde convocó a distintas personas a dialogar en torno a estas temáticas. Uno de los expositores invitados fue el profesor de Harvard, Frank Sander, a quien se reconoce en Estados Unidos como el inspirador o creador del modelo del Tribunal Multi-puertas ${ }^{49}$.

Sobre la base de una proyección realizada un año antes por el profesor John Barton, que auguraba una enorme alza de casos para los caurenta años siguientes, Frank Sander señala que es posible abordar este problema de dos formas. Una es tratando de prevenir conflictos haciendo cambios en las leyes sustantivas (lo que él llama un derecho preventivo) y, la otra, es explorando formas alternativas de resolver las disputas fuera de los tribunales ${ }^{50}$.

Asimismo, observa ya en la década de 1970 un fenómeno perfectamente extrapolable al Chile del siglo Xxi: un incremento progresivo de los casos ante los tribunales en múltiples áreas producto de la complejización de las sociedades modernas y del crecimiento del Estado, generando, con ello, el surgimiento de muchas nuevas fuentes de potenciales conflictos y las consecuentes expectativas por parte de los ciudadanos. Considera que en este nuevo escenario los tribunales no pueden responder efectivamente a la acelerada y creciente demanda y, por tanto, se hace esencial explorar otras alternativas $^{51}$, pues, a su juicio, el tiempo en que los tribunales eran

${ }^{46}$ Por ejemplo, el programa multi-puertas del distrito de Columbia ofrece a las partes mediación, arbitraje, conciliación y evaluación del caso (case evaluation) y ofrece enlaces con otros servicios relacionados en una amplia gama de campos, como materia civil, pequeñas causas y familia. Disponible en www.dccourts.gov/internet/superior/org_multidoor/main. jsf. [Fecha de consulta: 26 de abril de 2017].

${ }^{47}$ Véase www.dccourts.gov/internet/superior/org_multidoor/main.jsf. [Fecha de consulta: 26 de abril de 2017].

${ }^{48}$ Singer (1996), p. 19.

${ }^{49}$ Ibid.

${ }^{50}$ SANDER (1976), pp. 111-112.

${ }^{51}$ Op. cit., p. 113. 
el principal "procesador" de conflictos ya pasó ${ }^{52}$ (el énfasis es nuestro). Aboga, entonces, por un panorama diverso y flexible de procesos de resolución de conflictos, donde particulares tipos de conflicto sean asignados a diferentes procesos -o a una combinación de ellos- de acuerdo con distintos criterios como, por ejemplo, la naturaleza de la disputa y la relación entre las partes ${ }^{53}$.

Lo novedoso de este planteamiento reside en la convergencia entre el mundo judicial y el mundo de los mecanismos alternativos bajo el paraguas institucional de los tribunales de justicia. No propone crear un sistema de solución de controversias paralelo al judicial sino, por el contrario, plantea que mecanismos como la negociación, la mediación o el arbitraje formen parte de la oferta estatal y sean promovidos y ofrecidos por los propios tribunales. Integra, así, estos mecanismos dentro del sistema, dándoles un reconocimiento que modifica el curso del movimiento ADR que hasta ese momento se venía dando en Estados Unidos, cuyos mecanismos operaban como reales alternativas al sistema público ${ }^{54}$.

Lo que se desprende de este modelo es un cambio en la concepción del papel de los tribunales. Es el propio sistema judicial el que estimula a las partes a analizar la posibilidad de utilizar algún mecanismo alternativo para resolver sus controversias, incluso, en forma previa al proceso ${ }^{55}$. De este modo, la ley alienta a los jueces a derivar casos a mecanismos alternativos ${ }^{56}$, quienes, además, tienen a su cargo la capacitación de los terceros (neutrals) que llevarán a cabo los respectivos procesos alternativos.

En una entrevista que el profesor Frank Sander diera en 2008, señala que la denominación que originalmente tenía en mente para su propuesta de exploración de otras alternativas era "comprehensive justice center" ${ }^{57}$. El nombre de Tribunal Multi-puertas que hoy conocemos nace como consecuencia del trabajo que luego de la Conferencia Pound comienza a realizar el Departamento de Justicia del gobierno del entonces presidente Jimmy Carter y, en particular, de la labor desplegada con posterioridad por la American Bar Association ${ }^{58}$.

${ }^{52}$ SANDER (1976), p. 126.

${ }^{53}$ Op. cit., p. 133.

${ }^{54}$ NúÑ̃z (2009), p. 45.

${ }^{55}$ Op. cit., p. 48.

${ }^{56}$ Phipps \& Savage (2003), p. 329.

${ }^{57}$ SANder \& Hernández (2008), p. 670.

${ }^{58}$ A fines de la década de 1970 este organismo crea un comité especial sobre esta temática, el que luego se transformaría en la Sección de Resolución de Disputas (Dispute Resolution Section) que existe hasta hoy, comienza a realizar seminarios anuales sobre estas materias y crea una revista especializada. Para mayor información, véase www. americanbar.org/groups/dispute_resolution.html. [Fecha de consulta: 20 de julio de 2018]. 
Años después, en 1983 se dictan las Federal Rules of Civil Procedure que establecen que uno de los propósitos de las pretrial conferences es precisamente facilitar los acuerdos entre las partes ${ }^{59}$. En 1984 se desarrolla un proyecto piloto de Tribunales Multi-puertas en tres Estados del país: Tulsa (Oklahoma), Houston (Texas) y Washington, D.C., experiencia piloto que marca el inicio de la puesta en marcha de la idea que el profesor Frank Sander propusiera a mediados de la década de 1970. Luego, en 1998 se dicta el Acta de Resolución Alternativa de Disputas (Alternative Dispute Resolution Act) que obliga a las cortes de distrito federal a adoptar normas locales que autoricen y promuevan el uso de formas variadas de resolución de controversias civiles ${ }^{60}$.

De ahí en adelante la expansión de los mecanismos alternativos -o del movimiento ADR como se conoce en Estados Unidos- ha sido permanente y vigorosa, sumándose a esta nueva mirada las facultades de Derecho, las organizaciones de base, escuelas y gremios, entre muchos otros ${ }^{61}$. En la actualidad el modelo de Tribunal Multi-puertas existe a lo largo de todo el país del norte e, incluso, se ha "importado" a otras naciones con sistemas culturales y procesales muy distintos a los de Estados Unidos como es Nigeria, compartiendo, sin embargo, los mismos objetivos ${ }^{62}$.

\section{Premisas Para EL Diseño De Un SISTEMA INTEGRAdo} DE RESOLUCIÓN DE CONFLICTOS CIVILES

La búsqueda de una relación equilibrada, de mutua colaboración y apoyo entre el mundo judicial y el de los mecanismos alternativos, supone romper con el paradigma que estos mecanismos, ya sea por su especialidad

${ }^{59}$ Rule 16. Pretrial Conferences; Scheduling; Management. (a) Purposes of a Pretrial Conference. In any action, the court may order the attorneys and any unrepresented parties to appear for one or more pretrial conferences for such purposes as: (1) expediting disposition of the action; (2) establishing early and continuing control so that the case will not be protracted because of lack of management; (3) discouraging wasteful pretrial activities; (4) improving the quality of the trial through more thorough preparation; and (5) facilitating settlement. Disponible en www.uscourts.gov/sites/default/files/rules-ofcivil-procedure.pdf. [Fecha de consulta: 30 de abril de 2017].

${ }^{60} 28$ U.S. Code $\$ 651$-Authorization of alternative dispute resolution. En el preámbulo de esta Acta, entre otras ideas, se establece que los MASC, como la mediación, evaluación neutral, mini juicios (minitrials) y el arbitraje voluntario tienen el potencial de traer de variados beneficios a la comunidad, como la satisfacción de las partes y mayor eficiencia en lograr acuerdos. Véase www.adr.gov/ADR\%20ACT\%201998.pdf. [Fecha de consulta: 3 de mayo de 2017].

${ }^{61}$ Singer (1996), p. 11-29.

${ }^{62}$ Véase www.lagosmultidoor.org.ng/welcome/. [Fecha de consulta: 26 de abril de 2017]. 
o requerimientos específicos, exigen una legislación ad hoc escindida de los códigos procesales ${ }^{63}$.

La tarea de un código procesal no es solo regular el proceso judicial y sus etapas, sino la de articular distintas modalidades de solución de controversias civiles y el tránsito coordinado entre ellos. Esta tarea de articulación requiere, desde luego, ciertas premisas básicas -algunas de las cuales se presentan a continuación- las que pueden servir como punto de partida para la discusión sobre cómo diseñar un sistema integral de resolución de conflictos civiles.

Es importante señalar que las proposiciones aquí planteadas no tienen pretensión de exhaustividad, pues un proceso de reforma en esta materia exige hacerse cargo de muchos otros -y muy relevantes- aspectos de diseño e implementación de una política pública, que escapan del objetivo de este trabajo. En efecto, aspectos relativos a los costos, asistencia gratuita, evaluación de los mecanismos y ejecución de los acuerdos, son algunos de los delicados temas que será necesario analizar si se toma una opción como la que aquí se propone.

Considero que el diseño de un sistema integral de solución de controversias civiles debe descansar al menos en las siguientes cuatro premisas básicas, a saber:

- Sistema de opción múltiple que contempla varios mecanismos con varios puntos de entrada y salida, es decir, un sistema no lineal.

- Los mecanismos alternativos pueden aplicarse tanto a un litigio ya ingresado al sistema como aquel que aún no ha comenzado.

- Sistema de opción voluntaria y forzada, ya sea a iniciativa de las partes, de derivación judicial o que se exija como un requisito de procesabilidad de la demanda.

- Lo avanzado en uno de los mecanismos sirve de punto de partida o precedente para el siguiente, salvo en el caso de un proceso de mediación.

A continuación, veremos cada una de ellas.

${ }^{63}$ En este punto vale la pena a traer a colación lo que establece la directiva 2008/52/ce del Parlamento Europeo y del Consejo sobre ciertos aspectos de la mediación en asuntos civiles y mercantiles transfronterizos, muestra claramente el enfoque que el Consejo de Europa ha definido en esta materia. El objetivo de esta directiva es: "facilitar el acceso a modalidades alternativas de solución de conflictos y fomentar la resolución amistosa de litigios promoviendo el uso de la mediación y asegurando una relación equilibrada entre la mediación y el proceso judicial". Art. 1. Directiva 2008/52/ce del Parlamento Europeo y del Consejo de 21 de mayo de 2008. Dsiponible en http://eur-lex.europa.eu/ LexUriServ/LexUriServ.do?uri=OJ:L:2008:136:0003:0008:Es:PDF. [Fecha de consulta: 27 de enero de 2018]. 


\section{Primera premisa: \\ Sistema de opción múltiple que contempla varios mecanismos con varios puntos de entrada y salida, es decir, un sistema no lineal}

El mundo de los mecanismos alternativos es amplio y diverso. En la experiencia comparada se advierte el desarrollo e institucionalización de una enorme variedad de dispositivos y combinaciones de ellos, algunos de los cuales son desconocidos en nuestro medio ${ }^{64}$. Por ello, esta propuesta recoge solo tres mecanismos alternativos -conciliación, mediación y arbitraje-, dado que forman parte del ordenamiento jurídico nacional y respecto de los cuales existe experiencia práctica que puede arrojar luces sobre cómo mejorar su aplicación y resultados.

En primer lugar, un sistema integral debe potenciar fuertemente la conciliación judicial. Para ello, los jueces deben comprender que forma parte de su papel alentar y promover los acuerdos amistosos entre las partes. Esto implica destinar ingentes esfuerzos en entregar a los jueces formación en destrezas y habilidades de negociación, parafraseo, escucha activa, entre otras. Pero lo más relevante es cambiar la cultura imperante e intentar que internalicen que la labor conciliatoria forma parte integrante de la labor judicial, que, si bien no es una función estrictamente jurisdiccional, es una de las tareas que se ha entregado a nuestros tribunales de justicia desde tiempos inmemoriales ${ }^{65}$.

Por otra parte, el sistema de gestión interno del tribunal debe dotar al juez de suficiente tiempo para llevar a cabo esta función -preferentemente por medio de una audiencia destinada exclusivamente a estos efectos- de modo que le permita hacer preguntas, explorar puntos de convergencia y proponer bases de arreglo. Debe dotarse al juez de la posibilidad de suspender ("congelar") el procedimiento si las partes requieren de tiempo para dialogar o pensar las propuestas $^{66}$ y de "dividir" el asunto, si es viable un acuerdo parcial. Adicionalmente, debe facultarse expresamente al juez para ofrecer o llamar a las partes a una instancia de conciliación cuantas veces sea necesario durante el proceso, pues a medida que este avanza las partes y el juez cuentan con mayor información acerca de los hechos, de las pruebas (probablemente el aspecto más relevante) y puede haber entonces mayor disposición para un acuerdo.

Para fortalecer aún más este mecanismo debiera exigirse que las partes comparezcan personalmente a la audiencia de conciliación, aun cuando

${ }^{64}$ Steffer y Unberath (2013), p. 41.

${ }^{65}$ Vargas (1996), pp. 15-114.

${ }^{66}$ Esta facultad se observa en la Ley 21081, que modifica la ley 19496 de Protección de Derechos de los Consumidores publicada en el Diario Oficial el 13 de septiembre de 2008. Allí, en el procedimiento judicial que se contempla para acciones de interés colectivo o difuso, se fortalece la labor de conciliación de los jueces civiles y se les permite suspender la audiencia de conciliación "para facilitar la deliberación de las partes". 
hayan otorgado a sus mandatarios la facultad de transigir, de modo de sentar en una misma mesa a los directamente afectados y analizar seriamente alternativas de solución. Una forma de incentivar esta participación es por la vía de suspender el procedimiento en caso de inasistencia de ambas partes, sin perjuicio de explorar otras fórmulas más drásticas como la condena en costas a aquel que no comparece con causa justificada, como se observa en el modelo inglés de mediación o de una presunción simple en contra del demandado respecto de los hechos alegados por el actor que contempla el Código General del Proceso de Uruguay en el caso de la conciliación previa ${ }^{67}$.

En segundo lugar, la mediación forma parte ineludible de la oferta de mecanismos alternativos que un sistema integral de resolución de conflictos civiles debiera proveer. En un sistema como el que se propone, la relación de los jueces con la mediación es de remisión o referencia, lo que se dará en cuatro momentos específicos:

a) evaluación de la factibilidad que un caso objeto de un proceso judicial pueda ser derivado a mediación;

b) derivación a mediación contado para ello con información apropiada y canales de comunicación expeditos y eficientes,

c) información sobre los resultados de la mediación, esto es, si hay acuerdos o no, si son totales o parciales y

d) ejecución de los acuerdos si una o ambas partes los incumplen ${ }^{68}$.

Como se observa, no se trata de entregar la conducción de la mediación a los jueces ni a los funcionarios judiciales, sino de crear un sistema de derivación directa de los casos a mediadores profesionales. La definición acerca de quiénes pueden ser mediadores y las exigencias que ellos deben cumplir es un aspecto tremendamente relevante y complejo. Ello depende de una serie de factores orgánicos, presupuestarios y de política pública para lo cual se puede tener presente la experiencia comparada ${ }^{69} \mathrm{y}$, desde luego, la nacional en esta materia ${ }^{70}$.

${ }^{67}$ En materia civil se regula la obligatoriedad de la tentativa de una conciliación previa a cargo de jueces especializados de conciliación o jueces de paz, según se trate de casos de competencia de tribunales de la capital o del interior, donde se prevén consecuencias para ambas partes en casos de inasistencia. Art. 295.3 del Código General del Proceso de Uruguay.

${ }^{68}$ En este punto se sigue el modelo de court-annexed mediation de Estados Unidos, que aquí se menciona muy sucintamente. Para mayor información, véase las normas federales en materia de programas de mediación anexa a tribunales del Departamento de Justicia de Estados Unidos, véase www.justice.gov/olp/federal-court-annexed-adr-programs-glance [fecha de consulta:18 de julio de 2018].

${ }^{69} \mathrm{La}$ experiencia estadounidense muestra variadas alternativas, por ejemplo, mediadores que formen parte del staff del Poder Judicial, mediadores pagados o probono e, incluso, exmagistrados que ejercen esta labor. Para mayor información, véase www.justice. gov/olp/federal-court-annexed-adr-programs-glance [fecha de consulta:18 de julio de 2018].

${ }^{70}$ Resulta imprescindible analizar, también, la experiencia nacional donde se observa una enorme variedad de modelos: mediadores privados licitados en familia (art. 112 inc. 
En tercer lugar, el arbitraje también debe ser considerado en este sistema integral de resolución de conflictos civiles. Se trata de un mecanismo de antigua data en nuestro ordenamiento jurídico y de gran utilización en materia civil y comercial, ya sea a través del arbitraje ad hoc o el arbitraje institucional que se realiza a través de centros especializados ${ }^{71}$.

$\mathrm{Al}$ igual que la mediación deben regularse en forma clara las conexiones del arbitraje con el proceso judicial, respetando la autonomía de cada uno de estos procedimientos en relación con su operación y funcionamiento. El papel del juez será, entonces, de remisión o referencia y debiera darse en los mismos términos antes mencionados:

a) evaluación de la factibilidad que un caso objeto de un proceso judicial pueda ser derivado a arbitraje;

b) derivación a arbitraje a través de canales expeditos y eficientes,

c) información sobre los resultados del arbitraje y

d) ejecución de los laudos arbitrales, si una o ambas partes lo incumplen.

En relación con el profesional a cargo de estos procesos, a diferencia de lo que ocurre en mediación, en este caso hay camino avanzado. Nuestro sistema judicial cuenta con registros de jueces árbitros elaborados por el Poder Judicial para las distintas jurisdicciones del país, a los cuales los jueces pueden derivar un asunto en los casos en que la ley lo establece. 212 Un aspecto cuya regulación parece medular es asegurar a las partes y a los profesionales un sistema de designación aleatorio, de modo de dotar de transparencia al mecanismo e incentivar la postulación a estos registros de profesionales bien calificados y con experiencia en el área.

primero, La Ley 19968, que crea los Tribunales de Familia, Decreto 933 y Decreto 763 que aprueba reglamento de la Ley n. ${ }^{\circ}$ 19968, que crea los tribunales de familia y deroga decreto n. ${ }^{\circ}$ 957, que aprueba normas reglamentarias necesarias para la ejecución de la ley n. ${ }^{\circ}$ 19968); mediadores del Consejo de Defensa del Estado para daños en salud si se trata de organismos públicos y mediadores de un registro de la Superintendencia de Salud si se trata de organismos privados (art. 43, Ley 19.966, que establece el Régimen de Garantías en Salud) y mediadores de la Dirección del Trabajo art. 486 inc. 6, Ley 20087, que sustituye el procedimiento laboral contemplado en el libro v del Código del Trabajo publicada en el Diario Oficial el 3 de enero de 2006).

${ }^{71}$ Uno de ellos es el Centro de Arbitraje y Mediación de la Cámara de Comercio de Santiago, que ofrece servicios de arbitraje y mediación para la resolución de controversias nacionales e internacionales, ha llevado más de tres mil casos de arbitraje en veinticinco años de funcionamiento. Véase www.camsantiago.cl/index.htm [fecha de consulta: 20 de julio de 2018). Otro centro especializado, en este caso, de arbitraje on line es el Centro de Resolución de Controversias por Nombres de Dominio.cl, el cual, a través de una plataforma electrónica, ofrece servicios de arbitraje para conflictos con ocasión de la revocación de un nombre de dominio. Véase www.nic.cl/controversias/el_centro.html. [Fecha de consulta: 20 de julio de 2018]. 


\section{Segunda premisa. Los mecanismos alternativos pueden aplicarse tanto a un litigio ya ingresado al sistema, como aquel que aún no ha comenzado}

Esta premisa nos lleva a una definición de carácter temporal. Se trata de determinar "cuándo" deben o pueden operar los mecanismos alternativos, si antes o durante el proceso judicial y, en este último caso, en qué etapa procesal específica.

En general, las opciones hasta ahora utilizadas han situado a estos mecanismos en momentos procesales determinados, especialmente cuando se trata de una exigencia obligatoria, sin margen de acción para acceder a ellos en otros momentos. Lo anterior podría explicar, entre otras cosas, la escasa aplicación y bajos resultados que algunos de estos han exhibido, como ocurre, por ejemplo, con la conciliación judicial ${ }^{72}$. Parece necesario, entonces, regular un sistema tanto de acceso como de derivación a estos mecanismos más flexible, que sin que caer en la informalidad, permita a las partes participar o al juez derivarlas en distintas etapas procesales.

Siguiendo con este enfoque se podría contemplar el acceso a estos mecanismos:

a) antes de la presentación de la demanda,

b) inmediatamente después de presentada la demanda,

c) una vez transcurrido un determinado plazo o etapa judicial definida ex ante

d) cuando el juez lo determine en cualquier estado del proceso y

e) cuando las partes lo soliciten en cualquier estado del proceso.

La definición del momento procesal apropiado para cada uno de ellos debe realizarse en función de ciertos criterios: como la naturaleza del asunto, su complejidad y el grado de adversarialidad que se observa entre las partes. De acuerdo con estos criterios, para algunas materias podría contemplarse la posibilidad de participar en un mecanismo alternativo en forma previa a la interposición de la demanda. Ello podría evaluarse para los juicios de cobro de pesos, que son típicamente de aquellos en que la intervención oportuna de un tercero puede resultar clave para acercar a las partes y lograr un acuerdo, ya sea a través de una mediación o una conciliación judicial.

Ahora bien, con independencia del momento procesal en que estos mecanismos se sitúen será necesario asegurar a los litigantes que su derecho a accionar, si no lo han hecho, o de retomar el proceso judicial, si este ya se ha iniciado, permanece intacto, pues los plazos para interponer

${ }^{72}$ Véase cita n. ${ }^{\circ} 2$ sobre resultados de la conciliación judicial en procedimiento ordinario y sumario en tribunales civiles de Santiago. 
una acción judicial quedan suspendidos durante el tiempo en que el mecanismo alternativo se haya desarrollado ${ }^{73}$.

\section{Tercera premisa.}

Sistema de opción voluntaria y forzada, ya sea a iniciativa de las partes, de derivación judicial o que se exija como un requisito de procesabilidad de la demanda

Uno de los principales dolores de cabeza de los policy makers al momento de incorporar mecanismos alternativos es definir "cómo deben operar", esto es, si hacer de ellos una instancia voluntaria u obligatoria para las partes. ¿Deben iniciarse solo a solicitud de las partes o es posible que el juez o la ley les exijan participar en una conciliación, en una mediación o en un arbitraje?

Este es un aspecto crucial del diseño de un sistema integral y flexible, pues una de las razones que se han dado para explicar, por ejemplo, por qué la mediación no se utiliza masivamente pese a las ventajas que de ella se predican, reside, precisamente, en que no se ha alcanzado un adecuado balance entre la opción voluntaria de aplicación de estos mecanismos y 214 los incentivos para que los litigantes participen en ellos ${ }^{74}$.

Esta discusión -aplicable a todos los mecanismos alternativos- habitualmente se enfoca desde la perspectiva de la mediación y, más específicamente, desde la perspectiva del principio de la voluntariedad. En virtud de este principio las partes tienen la libertad para participar en una mediación ya sea si surge de iniciativa propia o si ello les ha sido sugerido $^{75}$. De este modo, la exigencia legal o judicial de participar en un proceso de mediación -cualquiera sea la forma en que esto se regulesupondría vulnerar este principio ${ }^{76}$.

Pero lo cierto es que en nuestro ordenamiento jurídico existen desde antaño instancias obligatorias o forzadas de aplicación de mecanismos alternativos, sin que se hubiesen rasgado vestiduras. Me refiero, por ejemplo, a la conciliación judicial que contemplan varios de nuestros procedimientos civiles, donde la ley exige al juez llamar a conciliación y a las partes asistir

${ }^{73}$ Véase https://e-justice.europa.eu/content_eu_overview_on_mediation-63-es.do. [Fecha de consulta: 27 de enero de 2016].

${ }^{74}$ De Palo y Trevor (2012), p. 6.

${ }^{75}$ Suares (2002), p. 30.

${ }^{76}$ Se han dado argumentos a favor y en contra de diversa índole de establecer la obligatoriedad tanto de la mediación como de otros mecanismos alternativos, tratándose de un punto no hay consenso. Véase, entre otros, a Aguirrezabal (2013), pp. 295-308 y VARGAS (2008), pp. 183-202. 
a ella ${ }^{77}$. Otra cosa es que no lo hagan o que habiendo asistido no se llegue a un acuerdo, pero conforme a la ley las partes tienen la obligación de hacerlo ${ }^{78}$. Algo similar ocurre con el arbitraje forzoso para ciertas materias según regula el Código Orgánico de Tribunales desde hace más de un siglo.

El punto aquí no es si se establece o no la obligación, lo importante es que ella no sea la única opción que se da los justiciables para resolver sus conflictos y se deje la puerta abierta para acudir a otros dispositivos cuando así lo deseen (el énfasis es nuestro). Se trata de dejar atrás la rigidez que ha primado en el abordaje de estas materias. El caso del arbitraje forzoso es sintomático de lo que se viene planteando, pues el legislador establece solo una vía y, además, de manera obligatoria para solucionar determinados asuntos, lo que limita seriamente el acceso a la justicia ${ }^{79} \mathrm{y}$ ha traído consecuencias muy negativas a quienes no pueden acceder a este mecanismo por razones económicas ${ }^{80}$.

Me parece que es posible diseñar un sistema que combine opciones voluntarias y obligatorias según ciertos factores previamente determinados, como la naturaleza y la complejidad del asunto. En los asuntos de pequeñas causas, por ejemplo, el tránsito por alguno de estos mecanismos debiera ser obligatorio, pudiendo el actor eximirse si ha participado en instancias extrajudiciales similares en instituciones privadas (como las clínicas jurídicas universitarias) o instancias pertenecientes a un organismo del Estado (como el SERNAC o la Corporación de Asistencia Judicial), según el caso.

Pero para que las partes consideren estos mecanismos como una real alternativa al proceso judicial, la labor de los jueces es clave. En efecto, un sistema de estas características está anclado en los jueces, en sus creencias, en sus prejuicios y en sus experiencias previas en este ámbito. Si los jueces han tenido éxito en la conciliación en casos pasados o si han visto disminuir el despacho por causa de la mediación es probable que tengan una actitud más abierta para promover el uso de mecanismos alternativos. $\mathrm{Si}$, por el contrario, los jueces visualizan esto como un trámite más, que les quita tiempo y no muestra buenos resultados, las posibilidades de implementación y de eficiencia de una propuesta como esta son muy bajas.

Ahora bien, sea que se otorgue a los jueces la facultad de derivar casos a un mecanismo alternativo o que ello surja de iniciativa de las partes,

${ }^{77}$ Vargas y Fuentes (2018), pp. 34-35.

${ }^{78}$ Art. 262 del Código de Procedimiento Civil.

${ }^{79}$ Una crítica a esta figura se puede analizar con detalle en JEQUIER (2011), pp. 453-498.

${ }^{80}$ Con el fin de paliar estas consecuencias, desde el año 2012 la Facultad de Derecho de la Universidad Diego Portales viene desarrollando un programa de arbitraje probono, junto con la Fundación Pro Bono, la Corporación de Asistencia Judicial de la Región Metropolitana y el Centro Nacional de Arbitraje. Para mayor información véanse: http://udptransparente. udp.cl/accion/ y www.cna.cl/arbitraje-pro-bono/ [Fecha de consulta: 12 de julio de 2018]. 
lo importante es garantizar a los litigantes que ellos no perderán desde ningún punto de vista la posibilidad de acudir al proceso judicial, ya que los plazos para interponer una acción judicial -tal como se mencionó anteriormente- quedan suspendidos durante el tiempo en que el respectivo mecanismo alternativo se haya desarrollado ${ }^{81}$.

\section{Cuarta premisa. \\ Lo avanzado en uno de los mecanismos sirve de punto de partida \\ o precedente para el siguiente, salvo en el caso de un proceso de mediación}

Esta premisa es probablemente de las más complejas, pues nos lleva a preguntarnos sobre el grado de confidencialidad de la información vertida en el marco de un procedimiento alternativo. La pregunta que hay que responder es la siguiente: $\mathrm{Si}$ el primer mecanismo empleado no prospera, ¿cuánta información es susceptible de ser transmitida al proceso judicial o a otros mecanismos alternativos que se desarrollan con posterioridad?

En este punto no hay dudas que la confidencialidad de los mecanismos alternativos debe ser garantizada a las partes por normas procesales específicas, con el fin de objetivo de permitir una mayor apertura a la hora de presentar sus puntos de vista y evaluar posibles soluciones ${ }^{82}$. Estas normas deben cubrir a todas las personas que se consideren relevantes en dicho procedimiento, desde luego a las partes y sus abogados, al tercero que conduce el proceso y eventualmente a algún experto que haya participado.

En este sentido, la ley procesal debe contemplar normas que otorguen a las partes y al tercero el derecho a no testificar en un juicio posterior y restricciones a la presentación de hechos y de pruebas obtenidas en un procedimiento alternativo ${ }^{83}$. Sin embargo, deben considerarse, también, límites a la confidencialidad, cuando sea necesario, particularmente en casos de protección de otras personas y prevención o detección de actos constitutivos de delitos ${ }^{84}$.

Ahora bien, la consideración de la confidencialidad como un principio rector de la mediación ha significado, a mi juicio, un freno a la hora de pensar cómo transitar de un mecanismo alternativo a otro sin vulnerar

${ }^{81}$ Véase https://e-justice.europa.eu/content_eu_overview_on_mediation-63-es.do.

[Fecha de consulta: 27 de enero de 2016].

${ }^{82}$ SuARes (2002), p. 36.

${ }^{83}$ Steffek \& Unberath (2013), p. 24.

${ }^{84}$ Una expresión de este tipo de límites se observa en el art. 105 de la Ley 19968 de Tribunales de Familia, que consagra expresamente que el mediador queda exento del deber de confidencialidad si toma conocimiento de situaciones de maltrato o abuso en contra de niños, niñas, adolescentes o discapacitados, debiendo dar a conocer a las partes el sentido de esta exención. 
este principio. Pero de nuevo, se tiende a analizar todos los mecanismos alternativos solo a la luz de uno de ellos. Si se piensa bien, en el caso del arbitraje y de la conciliación judicial ese deber de confidencialidad es más bien limitado, pues las partes pueden voluntariamente transmitir lo allí discutido en instancias posteriores, a menos que hayan pactado expresamente lo contrario en el caso de un arbitraje o que el juez pidiera reserva sobre algún punto en el caso de una conciliación judicial.

Me parece que cuando las partes han avanzado en la discusión, cuando han zanjado algunos puntos o han alcanzado acuerdos parciales, desde una perspectiva de eficiencia del sistema, no parece sensato tirar todo ese trabajo por la borda y comenzar de nuevo desde cero. Es cierto que esto entraña algunos peligros, por ello es indispensable regular la confidencialidad con claridad y determinar qué aspectos puntuales pueden excepcionalmente ser transmitidos, los que debieran reducirse solo a un reporte general y sucinto de los temas tratados y de los avances logrados, si los hubiere.

\section{Conclusiones}

En un contexto de reforma a la justicia civil como el que se retomará prontamente parece necesario abrirse a la posibilidad de explorar nuevas visiones sobre cómo diseñar sistemas de resolución de conflictos amplios y diversos, que permitan a los justiciables acceder al mecanismo que mejor se ajuste a sus necesidades y eventualmente transitar hacia distintas opciones. Todo ello, desde luego, regulado en forma coherente y que resguarde los derechos de las personas que han intervenido.

La imagen de la justicia de doble hélice propuesta por Neil Andrews rompe con el paradigma de que el proceso judicial es la única forma de resolver los conflictos de relevancia jurídica y cualquier otro mecanismo es considerado como anormal. A partir de esta idea es posible imaginar un sistema de justicia civil que integre mecanismos alternativos que convivan y se desarrollen en una relación de equilibrio con el proceso judicial sin hacerlos competir ni presentarlos como opciones excluyentes.

Algunos puntos de partida para iniciar este debate se resumen en las cuatro premisas básicas que se desarrollan en este documento:

a) sistema de opción múltiple que contempla varios mecanismos con varios puntos de entrada y salida, es decir, un sistema no lineal;

b) los mecanismos alternativos pueden aplicarse tanto a un litigio ya ingresado al sistema como aquel que aún no ha comenzado;

c) sistema de opción voluntaria y forzada, ya sea a iniciativa de las partes, de derivación judicial o que se exija como un requisito de procesabilidad de la demanda y 
d) lo avanzado en uno de los mecanismos sirve de punto de partida o precedente para el siguiente, salvo en el caso de un proceso de mediación.

Sin duda, muchas otras premisas podrían ser incorporadas en este debate. Desde luego, parece relevante detenerse en un asunto estructural que tiene relación con el modelo orgánico que ha sustentar un sistema de estas características o las formas de dar seguimiento y cumplimiento a los acuerdos alcanzados. A ello, se suman aspectos de infraestructura, costos, asesoría jurídica, entre otros, los cuales merecen una discusión aparte.

La reapertura de la discusión del proyecto de ley NCPC que propicia el gobierno del presidente Sebastián Piñera abre una ventana de oportunidad para repensar este tema de cara a la reforma a la justicia civil. No solo habría que repensar cómo optimizar el proceso judicial, cómo hacerlo de mejor calidad y más expedito, sino, también, se hace necesario repensar si valdría la pena correr las fronteras y dar un salto verdaderamente cualitativo en el diseño de nuestros sistemas de justicia civil para los próximos cien años que integre diversos mecanismos de solución de controversias y que regule el adecuado tránsito entre ellos.

Aguirrezábal, Maite; Pérez Ragone, Álvaro y Vargas, Macarena (2011). "Informe del XIV Congreso Mundial de la Asociación Internacional de Derecho Procesal: el justo proceso en examen (Procedural Justice)". Ius et Praxis. n. ${ }^{\circ}$ 2. Talca.

Aguirrezabal, Maite (2013). "Mediación previa obligatoria y acceso a la justicia en el proceso de familia”. Revista Chilena de Derecho Privado, $\mathrm{N}^{\circ} 20$. Santiago.

Andrews, Neil (2003). Justicia civil inglesa. Proceso y otras formas de resolución de controversias. Bogotá: Editorial Temis.

CAPPELletti, Mauro y Bryant GaRTH (1996). El acceso a la justicia. La tendencia en el movimiento mundial para hacer efectivos los derechos. México: Fondo de Cultura Económica.

De Palo, Guiseppe \& Mary Trevor B. (2012). EU Mediation. United Kingdom: Oxford University Press.

Fiss, Owen (1984). "Against the settlement". The Yale Law Journal. n. ${ }^{\circ}$ 6. New Heaven.

Genn, Hazel (2013). "What is Civil Justice For?. Reform, ADR and Access to Justice". Yale Journal of Law \& the Humanities. Volume 18. New Heaven.

GonZÁlez, Felipe (2007). Informe sobre acceso a la justicia en Iberoamérica. Proyecto realizado para la SEGIB y para el Programa de Naciones Unidas para el Desarrollo-PNUD. Madrid. 
JEQuier Lehuedé, Eduardo (2011). "El arbitraje forzoso en Chile (un examen de constitucionalidad en el ordenamiento jurídico chileno)". Estudios Constitucionales. Vol. 9. Santiago.

NúÑEz OJEDA, Raúl (2009). Negociación, mediación y conciliación como métodos de resolución de controversias. Santiago: Ediciones Jurídicas Santiago.

Phipps Senft, Louise \& Cynthia A. Savage (2003). "ADR in the courts: progress, problems and posibilities". Penn State Law Review Vol. 108. Dsiponible en www.courts.state.co.us/userfiles/File/Administration/Policy_and_Program/ ODR/documents/ADR $\% 20$ in $\% 20$ the $\% 20$ Courts $\% 20$ Progress $\% 20$ Problems $\% 20$ Possibilitites.pdf. [Fecha de consulta: 20 de julio de 2018].

Riego, Cristian y Ricardo Lillo (2014). "Las unidades de justicia vecinal en Chile y sus modelos en la experiencia de los Estados Unidos de Norteamérica”. Revista de Derecho de la Pontificia Universidad Católica de Valparaíso. n. ${ }^{\circ} 43$. Valparaíso.

SANDER, Frank (1976). "Varieties of Dispute Processing. National Conference on the Dissatisfaction with de Administration of Justice". Federal Rules Decisions. n. ${ }^{\circ} 1$. Minnesota.

Sander, Frank \& Mariana Hernández Crespo (2008). "A dialogue between professors Frank Sander and Mariana Hernández Crespo: Exploring the evolution of the multi-door courthouse". University of Saint Thomas Law Journal. Vol. 5. Miami.

Singer, Linda (1996), Resolución de conflictos: Técnicas de actuación en los ámbitos empresarial y legal. Barcelona: Editorial Paidós.

Steffek, Felix \& Hannes Unberath, (eds.) (2013). Regulating dispute resolution, $A D R$ and access to justice al the crossroads. United Kingdom: Hart Publishing.

SuAres, Marinés (2002), Mediando conflictos familiares. Buenos Aires: Editorial Paidós, Argentina.

Vargas, Macarena y Claudio Fuentes (2018). Introducción al Derecho Procesal. Nuevas aproximaciones. Santiago: DER Ediciones.

VARGAS, Macarena (2008). "Mediación obligatoria. Algunas razones para justificar su incorporación”. Revista de Derecho. Vol. XXI. n. ${ }^{\circ}$ 2. Valdivia.

VARGAS, Macarena (1996). "Las tratativas conciliatorias como gestiones preparatorias en el procedimiento civil". Cuadernos de Análisis Jurídico Reforma Procesal Civil II. n. ${ }^{\circ}$ 32. Santiago: Alfabeta impresores.

\section{Normas citadas}

Auto acordado 128. Corte Suprema. Instructivo para el registro y designación de jueces árbitros por parte de los tribunales ordinario. 17 de agosto de 2015 .

CRP. Pre-action protocols. Disponible en www.justice.gov.uk/courts/procedurerules/civil/rules/pd_pre-action_conduct. [Fecha deconsulta: 3 de abril de 2017. Directiva 2008/52/ce del Parlamento Europeo y del Consejo sobre ciertos aspectos de la mediación en asuntos civiles y mercantiles transfronterizos. 
Ley 19966, que establece el Régimen de Garantías en Salud publicada en el Diario Oficial el 3 de septiembre de 2004.

Ley 19968, que crea los Tribunales de Familia publicada en el Diario Oficial el 30 de agosto de 2004.

Ley 19971 de Arbitraje Comercial Internacional publicada en el Diario Oficial el 29 de septiembre de 2004.

Ley 20087, que contempla el nuevo procedimiento de tutela de derechos fundamentales publicada en el Diario Oficial el 3 de enero de 2006.

Ley 20286, que introduce modificaciones orgánicas y procedimentales a la Ley 19.968 de Tribunales de Familia publicada en el Diario Oficial el 15 de septiembre de 2008.

Ley 21.081, que modifica la Ley 19496 de Protección de Derechos de los Consumidores publicada en el Diario Oficial el 13 de septiembre de 2008.

Mensaje de S. E. El Presidente de la República, con el que se inicia un proyecto de ley que establece el nuevo código procesal civil.

\section{Informes}

Centro de Estudios de Justicia de las Américas (2011). Informe Final. Estudio de Análisis de Trayectoria de las causas civiles de los Tribunales Civiles de Santiago. $220 \quad$ Santiago.

Centro de Estudios de Justicia para las Américas (2016). Guía para la implementación de mecanismos alternativos al proceso judicial para favorecer el acceso a la justicia, Marco Fandino (coord.). Santiago: Mito Impresores.

Lord Woolf, Her Majesty's Stationary Office (1996). Access to Justice: Final report to the Lord Chancellor on Civil Justice in England and Wales.

Programa de Naciones Unidas para el Desarrollo- PNUD (2005). Manual de políticas públicas para el acceso a la justicia. América Latina y El Caribe. Buenos Aires: Ediciones del Instituto.

\section{Páginas web}

https://e-justice.europa.eu/content_eu_overview_on_mediation-63-es.do. [Fecha de consulta: 27 de enero de 2016].

www.justice.gov/olp/federal-court-annexed-adr-programs-glance [Fecha de consulta: 18 de julio de 2018].

www.camsantiago.cl/index.htm. [Fecha de consulta: 20 de julio de 2018].

www.nic.cl/controversias/el_centro.html. [Fecha de consulta: 20 de julio de 2018]. 